\title{
A Shape-Factor Method for Modeling Parallel and Axially-Varying Flow in Tubes and Channels of Complex Cross-Section Shapes
}

\author{
Mario F. Letelier and Juan S. Stockle \\ University of Santiago of Chile, \\ Chile
}

\section{Introduction}

In the study of some industrial, biological and natural fluidic systems it is often necessary to model fluid flow through tubes, channels or passages of complex geometries. The complexity may arise from the cross-sectional shape, or from longitudinal cross-section variation, or from both. Typical cases include flow of molten metals or plastics through dies and moulds, blood flow, microfluidic applications, and flow in porous media, among many others. Characteristics of these flows are laminar state, incompressibility, small rates of flow and varied time patterns. One field where pertinent applications are being developed at a fast rate is Microfluidics (Cetin and Li., 2008; Chen et al., 2008; Forte et al., 2008; Gebauer and Bocek, 2002 ; Mathies and Huang, 1992; Sommer et al., 2008; Srivastava et al., 2005; Woolley and Mathies, 1994; Yeger et al., 2006.) . In this specific field, present microchannel manufacturing techniques produce typically non-circular capillaries (Sommer et al., 2008). Also the introduction of electrical or magnetic field induce plastic behavior in the working fluid.

In particular, it is well known that blood is a biological fluid that behaves as a Newtonian fluid in arteries, veins and large capillaries, but becomes non-Newtonian in the smaller vessels, where the size of suspended particles is big as compared to the vessel's diameter size (Pedley, 2008). A relevant problem in this field as to the method presented in the next sections is the analysis of diseased arteries and veins for quirurgical interventions. Specifically, stenosed arteries are blood conduits of irregular geometry in which crosssection geometry usually varies along the vessel length.

The above context implies that it is desirable, particularly for modeling and design purposes, to count with analytical techniques that can integrate variables such as the noncircular cross-section of conduits, axial variation of conduit geometry, and plastic flow in some cases.

In this chapter it is presented a method of analysis that allows to address in a general way the problem here outlined.

The standard analytical technique for tube flow problems is usually the search of specific solutions to the momentum equations with associated boundary and initial conditions (Batchelor, 2000). Otherwise numerical solutions are developed for some purposes (Xue et al.,1995). 
The main aim of this chapter is, thus, to introduce and explore the potential use of a general analytical approach to irregular conduit flow, which makes it possible to determine velocity field, rate of flow, shear stress, recirculation regions and plug zones, this last when fluid plasticity is operant.

The method already referred to has been developed by the authors through specific applications mainly during the past decade. In this chapter some previous results are organized within a common analytical pattern, together with novel material.

This chapter includes sections for the general model, considering one velocity component and more than one velocity component versions, applications related to flow in straight tubes and to axially-varying flows, and a closing conclusion section.

\section{The general model}

The concept of "shape factor" herein used is applied to a function $G$ of spatial coordinates, such as when $G=0$, a series of closed curves are determined for a range of some parameters contained in $G$. One typical example is

$$
G=1-r^{2}+\varepsilon r^{n} \sin n \theta
$$

In this $(r, \theta)$ are polar coorfinates, $n$ is an integer number and $\varepsilon$ is a parameter such as that for $\varepsilon=0$ the curve described by (1) is a circle, and as $\varepsilon$ increases, the shape evolves to some limiting shape, controlled by $n$. In all cases here considered, the maximum allowable value of $\varepsilon$ is less than unity, and beyond that value, the curve is no longer a closed one. If $\varepsilon=\varepsilon_{c}$ is the critical, or maximum, allowable value of $\varepsilon$, then for the shape factor described by $(1), \varepsilon_{c}$ is found to be

A more general shape factor is

$$
\varepsilon_{c}=\frac{2}{n}\left(\frac{n-2}{n}\right)^{(n-2) / 2}
$$

\begin{tabular}{|l|c|}
\hline$n$ & $\varepsilon_{c}$ \\
\hline 3 & 0,385 \\
\hline 4 & 0,250 \\
\hline 5 & 0,186 \\
\hline 6 & 0,148 \\
\hline
\end{tabular}

$$
G=1-r^{2}+\varepsilon_{1} r^{n_{1}} \sin n_{1} \theta+\varepsilon_{2} r^{n_{2}} \sin n_{2} \theta+\cdots
$$

which leads to more complex shapes. Some instances of these shapes are shown in Fig. 1.

For the purposes of this presentation, a general shape factor in polar coordinates can be defined as

$$
G=h_{0}(r)+\varepsilon_{1} h_{1}(r, \theta)+\varepsilon_{2} h_{2}(r, \theta)+\cdots
$$

in which $h_{1}, h_{2} \ldots$ are boundary perturbation functions. For the case of channel flow, the structure of (4) may be the same, in which polar coordinated may be substituted by Cartesian coordinates. The specific characteristics of functions $h_{i}$ are determined by the nature of the equations of motion and associated boundary conditions.

Two relevant cases can be highlighted, namely, flow with one velocity component, and flow with more than one velocity component. 


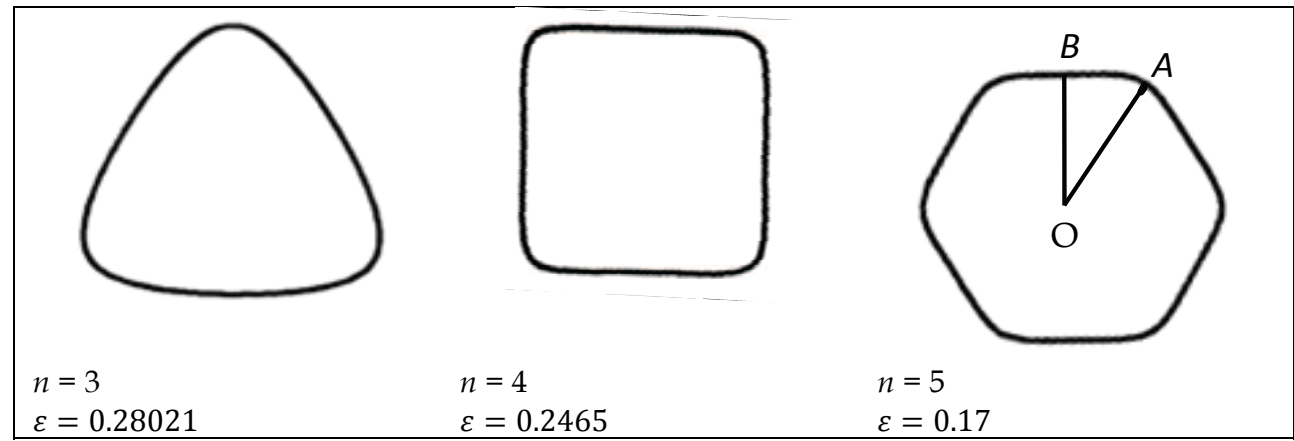

\begin{tabular}{|c|c|c|}
\hline $\begin{array}{l}n_{1}=3 \\
\varepsilon_{1}=0.113 \\
n_{2}=5 \\
\varepsilon_{2}=0.078\end{array}$ & $\begin{array}{l}n_{1}=2 \\
\varepsilon_{1}=0.258 \\
n_{2}=4 \\
\varepsilon_{2}=0.149\end{array}$ & $\begin{array}{l}n_{1}=2 \\
\varepsilon_{1}=0.21 \\
n_{2}=3 \\
\varepsilon_{2}=0.12\end{array}$ \\
\hline
\end{tabular}

Fig. 1. Samples of tubes contours from shape factors (1) and (3).

\subsection{Flow with one velocity component}

These are flows in straight tubes of constant cross-section. In these cases the axial velocity $w$ can be modeled as

$$
w(r, \theta, t)=G(r, \theta)\left[f_{0}(r, t)+\varepsilon f_{1}(r, \theta, t)+\varepsilon^{2} f_{2}(r, \theta, t)+\cdots\right]
$$

where, for the sake of simplicity, only one boundary perturbation function has been considered. Functions $f_{i}$ are to be determined from the equation of motion in terms of a standard regular perturbation scheme around the small parameter $\varepsilon$.

\subsection{Flows with more than one velocity component}

These are mainly flows with axial variation of tube or channel geometry. In these cases the solution procedure will usually involve the use of a stream function $\Psi$. In such problems both $\Psi$ and the velocity components should be zero at the boundary, a condition that can be met by defining

$$
\Psi=G^{2}\left[g_{0}(r, t)+\varepsilon g_{1}(r, \theta, t)+\varepsilon^{2} g_{2}(r, \theta, t)+\cdots\right]
$$

where again functions $g_{1}$ have to be determined from the equations of motion. The definition of $\Psi$ is given for every specific application in the corresponding section.

In the following some specific applications of this method of analysis are presented. 


\section{Applications}

\subsection{Flow in straight tubes of constant non-circular cross-section}

\subsubsection{Newtonian unsteady flow}

For incompressible, developed and isothermal flow, the equation of motion are the standard Navier-Stokes and continuity equations. In dimensionless variables they are

$$
\begin{gathered}
\Omega \frac{\partial w}{\partial t}-\left(\frac{1}{r} \frac{\partial w}{\partial r}+\frac{\partial^{2} w}{\partial r^{2}}+\frac{1}{r^{2}} \frac{\partial^{2} w}{\partial \theta^{2}}\right)=\phi(t)=-\frac{\partial P}{\partial z}(t) \\
\frac{\partial w}{\partial z}=0
\end{gathered}
$$

In this

$$
\Omega=\frac{\rho a^{2}}{\mu \mathrm{T}_{0}}
$$

is the so-called unsteadiness number of the flow, which measures the relative importance of a temporal inertia force against a steady viscous force, and where $\rho=$ density, $\alpha=$ reference tube radius, $\mu=$ dynamic viscosity, and $T_{0}=$ reference time.

A convenient solution of (7) (Letelier et al., 1995) for round tubes (ie for $\partial / \partial \theta=0$ ) can be worked out by postulating

$$
w=A_{2}\left(1-r^{2}\right)+A_{4}\left(1-r^{4}\right)+A_{6}\left(1-r^{6}\right)+\cdots
$$

Where $A_{2 n}=A_{2 n}(t)$ for $n=1,2,3 \ldots \infty$. Equation (10) meets the no-slip boundary condition $w(1, t)=0$. After substituting (10) in (7) it is found that all functions $A_{2 n}$ can be expressed in terms of $A_{2}=A$ so that the axial velocity takes the form

$$
w_{0}=\left(1-r^{2}\right)\left\{A+\frac{\Omega}{4^{2}} \frac{d A}{d t}\left(1+r^{2}\right)+\frac{\Omega^{2}}{4^{2} 6^{2}} \frac{d^{2} A}{d t^{2}}\left(1+r^{2}+r^{4}\right)+\cdots\right\}
$$

where $A$ is related to the forcing function $\phi(t)$ as follows

$$
\frac{\phi}{4}=A+\frac{\Omega}{2^{2}} \frac{d A}{d t}+\frac{\Omega^{2}}{2^{2} 4^{2}} \frac{d^{2} A}{d t^{2}}+\cdots
$$

In these expressions $\Omega$ can have any finite positive value.

According to (5), it is found

$$
f_{0}=A+\frac{\Omega}{4^{2}} \frac{d A}{d t}\left(1+r^{2}\right)+\frac{\Omega^{2}}{4^{2} 6^{2}} \frac{d^{2} A}{d t^{2}}\left(1+r^{2}+r^{4}\right) \cdots
$$

If

$$
\mathrm{L}=\Omega \frac{\partial}{\partial \mathrm{t}}-\left(\frac{1}{\mathrm{r}} \frac{\partial}{\partial \mathrm{r}}+\frac{\partial^{2}}{\partial \mathrm{r}^{2}}+\frac{1}{\mathrm{r}^{2}} \frac{\partial^{2}}{\partial \theta^{2}}\right)
$$

then, by collecting terms of order $\varepsilon$, it follows

$$
L\left\{f_{1}\left(1-r^{2}\right)\right\}=L\left\{f_{0} r^{n} \sin (n \theta)\right\}
$$

wherefrom

$$
f_{1}=\sin (n \theta) \sum_{i=1}^{\alpha}\left\{\Omega^{\mathrm{i}} \frac{\mathrm{d}^{\mathrm{i}} \mathrm{A}}{\mathrm{dt}}\right\} \sum_{s=0}^{2 i-2}\left\{C_{i s} r^{i+s}\right\}
$$


The constants $C_{i s}$ in these equations are obtained by putting the coefficients of all powers of $r$, for any $d^{i} A / d t^{i}(i=1,2, \ldots)$,equal to zero in (13). The result is

$$
\begin{gathered}
C_{10}=\frac{n-3}{16(n+1)} \\
C_{22}=\frac{72 C_{10}+n-2.5}{576(n+2)} \\
C_{20}=\frac{(n+1)\left(576 C_{22}+1\right)-144 C_{10}-9}{576(n+1)}
\end{gathered}
$$

and so on. Higher order terms in $\varepsilon$ can be obtained in like fashion.

An example of velocity profiles is shown in figure 1 for $n=6, \varepsilon=0.148$ at two semi-axes (cf Fig. 1).

In this case the tube contour is an approximate hexagon and $\phi=c o s t$, ie a purely oscillatory flow is described.

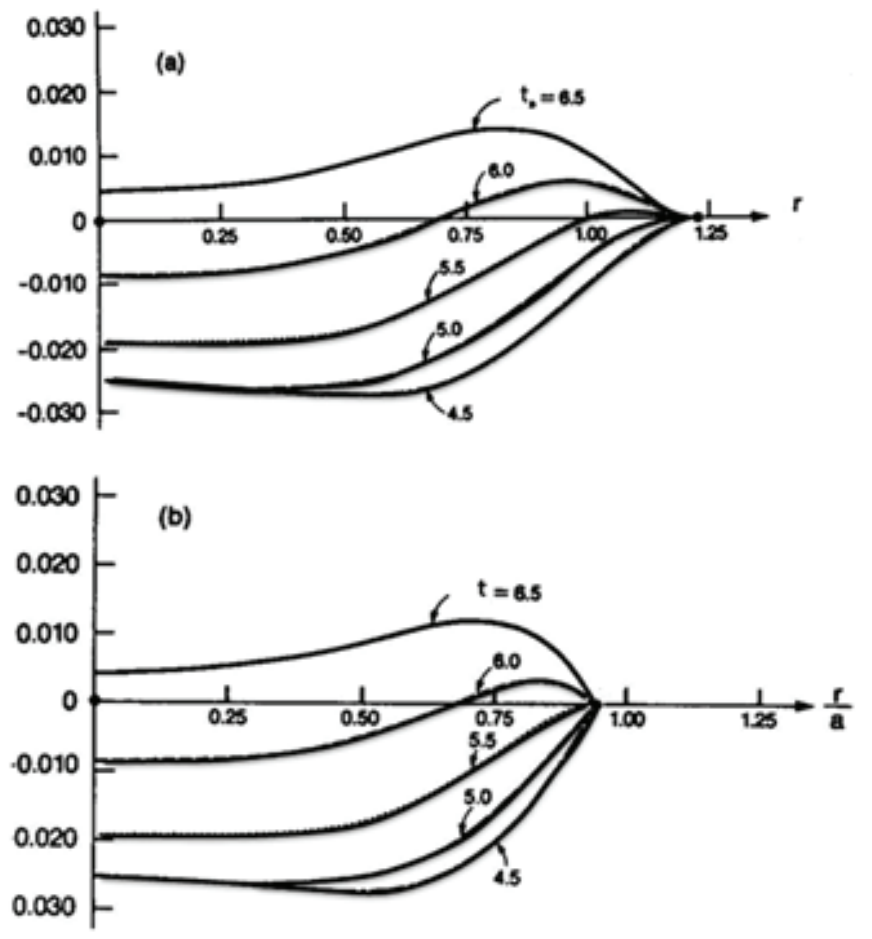

Fig. 2. Instantaneous velocity profiles for $\Omega=40$. (a) along maximum semi-axis OA; (b) along minimum semi-axis OB.

The structure of (4) makes it possible to apply a regular perturbation method of solution around the dimensionless parameter $\varepsilon$. Since $\varepsilon$ is bounded for a given value of $n$, and is always less than unity, the solution becomes actually an exact one when enough terms are obtained. 


\subsubsection{Steady plastic flow}

In this application it is considered steady flow of a Bingham plastic. Here (5) is also applicable, and the equation of motion, in terms of shear stress, as defined below, is

$$
\begin{gathered}
\frac{\tau_{r z}}{r}+\frac{\partial \tau_{r z}}{\partial r}+\frac{1}{r} \frac{\partial \tau_{\theta z}}{\partial \theta}=\phi \\
\tau_{r z}=-\left(1-\frac{N}{I}\right) \frac{\partial w}{\partial r} \\
\tau_{\theta z}=-\left(1-\frac{N}{I}\right) \frac{1}{r} \frac{\partial w}{\partial \theta}
\end{gathered}
$$

and

$$
I=\sqrt{\left(\frac{\partial w}{\partial r}\right)^{2}+\left(\frac{1}{r} \frac{\partial w}{\partial \theta}\right)^{2}}
$$

is the second invariant of the rate of deformation tensor. The dimensionless yield stress is

$$
N=\frac{\tau_{0} a}{w_{0} \eta_{0}}
$$

The momentum equation (20) is the standard one for parallel steady flow. Its structure has been made consistent with (21-22) and with the standard mathematical ordering of terms. The constitutive expressions (21-22) come from the applicable form of the Bingham fluid model. Defining

$$
w=w_{0}+\varepsilon w_{1}+\cdots
$$

then, from (5) it follows

$$
\begin{gathered}
w_{0}=\left(1-r^{2}\right) f_{0} \\
w_{1}=\left(1-r^{2}\right) f_{1}+r^{n} f_{0} \sin n \theta
\end{gathered}
$$

and the following equations are found

$$
\begin{gathered}
\tau_{r Z}=N-\frac{\partial w_{0}(r)}{\partial r}-\varepsilon \frac{\partial w_{1}(r, \theta)}{\partial r} \\
\tau_{\theta Z}=\left(\frac{N}{\left.\frac{\partial w_{0}(r)}{\partial r}-1\right) \frac{\varepsilon}{r} \frac{\partial w_{1}(r, \theta)}{\partial \theta}}\right. \\
-\frac{1}{r} \frac{\partial w_{0}(r)}{\partial r}-\frac{\partial^{2} w_{0}(r)}{\partial r^{2}}=\phi-\frac{N}{r} \\
\frac{1}{r} \frac{\partial w_{1}(r, \theta)}{\partial r}+\frac{\partial^{2} w_{1}(r, \theta)}{\partial r^{2}}+\left(1-\frac{N}{\frac{\partial w_{0}(r)}{\partial r}}\right) \frac{1}{r^{2}} \frac{\partial^{2} w_{1}(r, \theta)}{\partial \theta^{2}}=0
\end{gathered}
$$

Equations (28-29) are the result of substituting (25) in (23) and of ordering terms in powers of $\varepsilon$ through a linearization procedure. From (30-31) it is found

$$
w_{0}(r)=N(r-1)+\frac{\phi}{4}\left(1-r^{2}\right)
$$




$$
w_{1}(r, \theta)=A_{0}\left(1+n^{2} \sum_{i=1}^{n} \frac{(-1)^{i} \phi^{i}\left(n^{2}-(i-1)^{2}\right) !}{2^{i} N^{i}\left(i^{2}\right) !} r^{i}\right) \cos (n \theta)
$$

where

$$
A_{0}=\frac{2^{n}\left[n^{2} !\right] N^{n}}{(-1)^{n} \phi^{n}\left[n^{2}\left(n^{2}-1\right)\left(n^{2}-2^{2}\right) \ldots\left(n^{2}-(n-1)^{2}\right)\right]}
$$

Functions $f_{0}, f$, and following can be found equating terms in orders of $\varepsilon$ in (5), ie

$$
\begin{gathered}
f_{0}=\frac{w_{0}}{1-r^{2}} \\
f_{1}=\frac{w_{1}-r^{n} f_{0} \sin n \theta}{1-r^{2}}
\end{gathered}
$$

In this both functions are continuous for $r=1$, and so can be built higher order functions. Isovel plots and plug zones for selected instances of flow are shown in figure 3. A plug zone is such that inside its limiting boundary the shear stress is less than the yield stress.

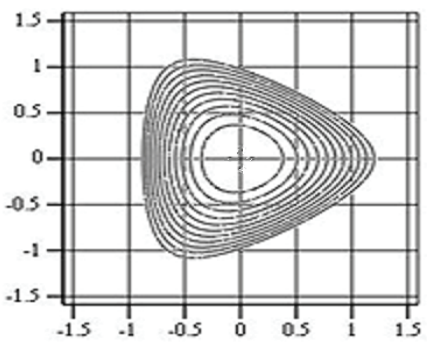

(a)

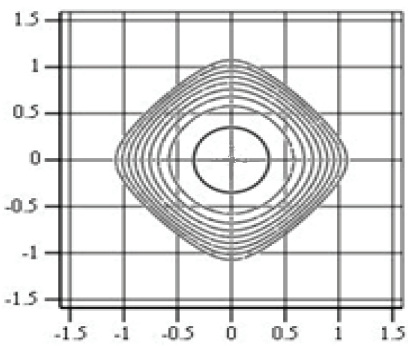

(b)

Fig. 3. Isovels from (25) for (a) $n=3, \varepsilon=0.3, N=0.2$ and $\phi=4$; (b) $n=4, \varepsilon=0.24$; $N=0.7$ and $\phi=4$.

According to fig.3, in both cases therein depicted, the plug zone appears at the center and is essentially circular.

\subsection{Axially-varying flows in conduits}

3.2.1 Newtonian flow in round tubes of arbitrarily axially-varying cross-section A definition diagram is shown in fig. 4

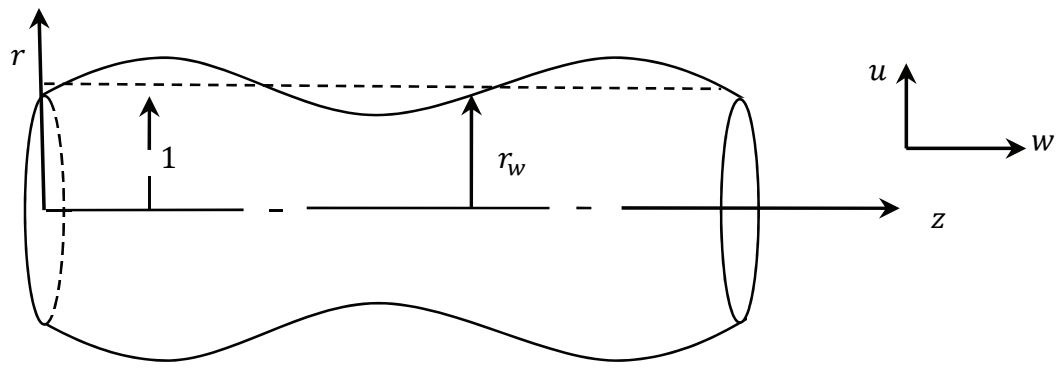

Fig. 4. Definitions diagram for flow in round tubes of axially-varying cross-section. 
For this flow, the continuity and Navier-Stokes equations are

$$
\begin{gathered}
\frac{1}{r} \frac{\partial(r u)}{\partial r}+\frac{\partial w}{\partial z}=0 \\
u \frac{\partial u}{\partial r}+w \frac{\partial u}{\partial z}=-\frac{2}{R e} \frac{\partial P}{\partial r}+\frac{2}{R e}\left(\frac{\partial}{\partial r}\left(\frac{1}{r} \frac{\partial(r u)}{\partial r}\right)+\frac{\partial^{2} u}{\partial z^{2}}\right) \\
u \frac{\partial w}{\partial r}+w \frac{\partial w}{\partial z}=-\frac{2}{R e} \frac{\partial P}{\partial z}+\frac{2}{R e}\left(\frac{1}{r} \frac{\partial}{\partial r}\left(r \frac{\partial w}{\partial r}\right)+\frac{\partial^{2} w}{\partial z^{2}}\right)
\end{gathered}
$$

where $R e$ is the Reynolds number. The velocity field is found after defining

$$
\begin{gathered}
u=\varepsilon u_{1}(r, z) \ldots \\
w=w_{0}(r)+\varepsilon w_{1}(r, z) \ldots \\
P=P_{0}(z)+\varepsilon P_{1}(r, z) \ldots \\
\Psi=\Psi_{0}(r)+\varepsilon \Psi_{1}(r, z) \ldots
\end{gathered}
$$

The velocity is next expressed in terms of de stream function as follows

$$
\begin{gathered}
w=\frac{1}{r} \frac{\partial w}{\partial r} \\
u=-\frac{1}{r} \frac{\partial \Psi}{\partial z}
\end{gathered}
$$

The wall radius is a function of the axial coordinate, that is here defined as

$$
r_{w}=1+\varepsilon h(r) F(z)
$$

in which $\varepsilon$ is a small parameter. This algebraic structure allows to introduce a very large range of axial variation since $h$ and $F$ are arbitrary functions. Three cases will be considered. In the first case

$$
h=r ; \quad F(z)=\sin \omega z
$$

where is $\omega$ is an arbitrary frequency.

The stream function is modelled from (6) and (46), ie

$$
\Psi=\left(r_{w}-r\right)^{2}\left[g_{0}(r)+\varepsilon\left\{g_{11}(r) \sin (\omega z)+g_{12}(r) \cos (\omega z)\right\}+0\left(\varepsilon^{2}\right)+\cdots\right]
$$

wherefrom

$$
\Psi_{0}=(1-r)^{2} g_{0}(r)=\frac{\phi}{16}\left(r^{2}-1\right)^{2}
$$

and thus

$$
g_{0}(r)=\frac{\phi}{16}
$$

The first order stream funtion is

$$
\Psi_{1}=(1-r)^{2}\left(g_{11}(r) \sin (\omega z)+g_{22}(r) \cos (\omega z)\right)+2(1-r) r \sin (\omega z) \frac{\phi}{16}
$$


which is next written as

$$
\Psi_{1}=\mathrm{H}_{1}(\mathrm{r}) * \sin (\omega \mathrm{z})+\mathrm{H}_{2}(\mathrm{r}) * \cos (\omega \mathrm{z})
$$

and where $H_{1}$ and $H_{2}$ are unknowns that are modelled as finite polynominals of even order terms, ie

$$
\begin{aligned}
& H_{1}(r)=a_{0}+a_{2} r^{2}+a_{4} r^{4}+a_{6} r^{6}+a_{8} r^{8}+a_{10} r^{10}+a_{12} r^{12}+a_{14} r^{14}+a_{16} r^{16} \\
& H_{2}(r)=b_{0}+b_{2} r^{2}+b_{4} r^{4}+b_{6} r^{6}+b_{8} r^{8}+b_{10} r^{10}+b_{12} r^{12}+b_{14} r^{14}+b_{16} r^{16}
\end{aligned}
$$

The coefficients $a_{i}$ and $b_{j}$ are determined by substituting (53) and (54) in the equation for $\Psi$ found from (38-39) once (44-45) are substituted in there. Examples of typical streamline and isovelocity patterns are shown in figures 5 and 6 . Streamlines are plotted from (48) and isovelocity are curves where $u^{2}+v^{2}$ is constant.

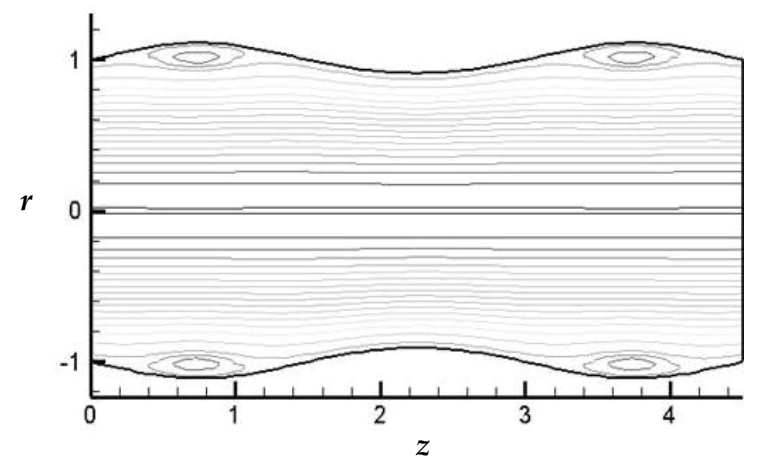

Fig. 5. Streamlines for $\operatorname{Re}=100$ and $\varepsilon=0.1$.

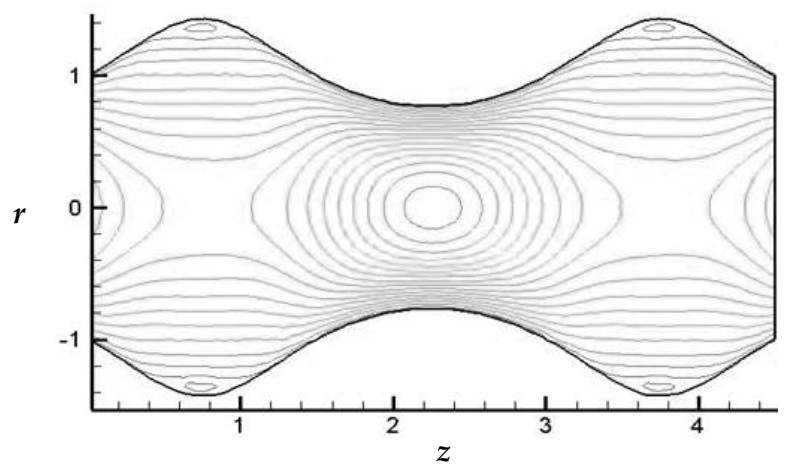

Fig. 6. Isovelocity lines for $\operatorname{Re}=1$ and $\varepsilon=0.3$ from (48) and (44-45).

A second kind of contour is defined through the expression

$$
F(z)=-0.028 z^{4}+0.434 z^{3}-2.156 z^{2}+3.43 z
$$


which was transformed in a Fourier series in the range $0 \leq z \leq 3$ in terms of sine and cosine functions that allow a modeling similar, but more complex, to that already described. Examples of typical streamline and isovelocity patterns are shown in figure 7 and 8.

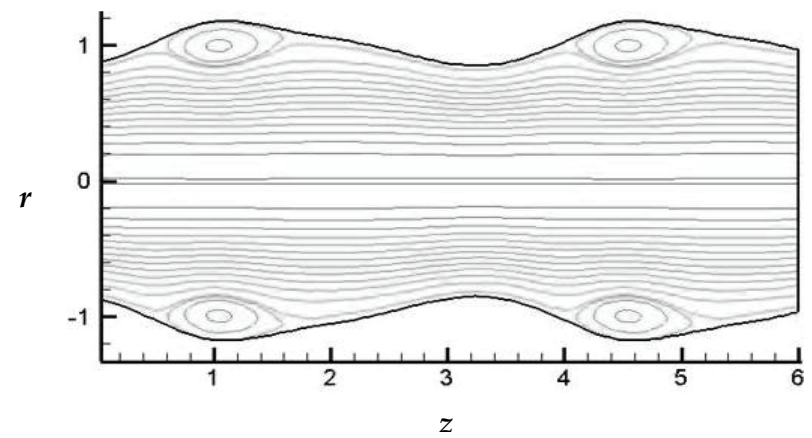

Fig. 7. Streamlines for $\operatorname{Re}=100$ and $\varepsilon=0.2$.

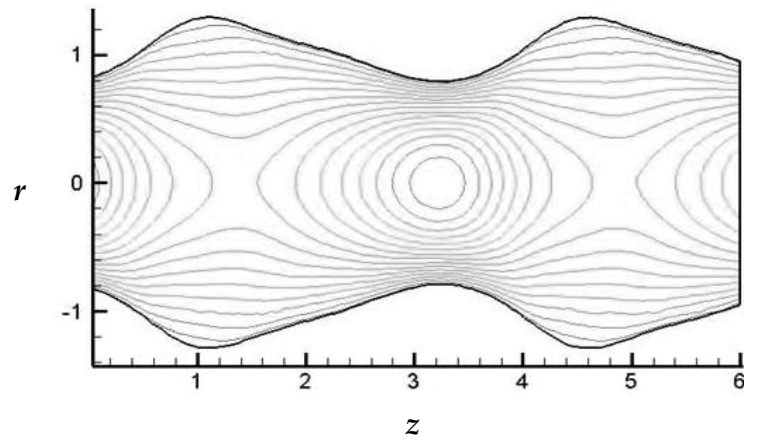

Fig. 8. Isovelocity lines for $\operatorname{Re}=1$ and $\varepsilon=0.3$.

In similar fashion, the a third contour presented is defined by

$$
F(z)=-0.03 z^{4}-0.045 z^{3}+0.405 z^{2}+0.42 z
$$

For this case, typical isovelocity and isobaric curves are shown in figures 9 and 10.

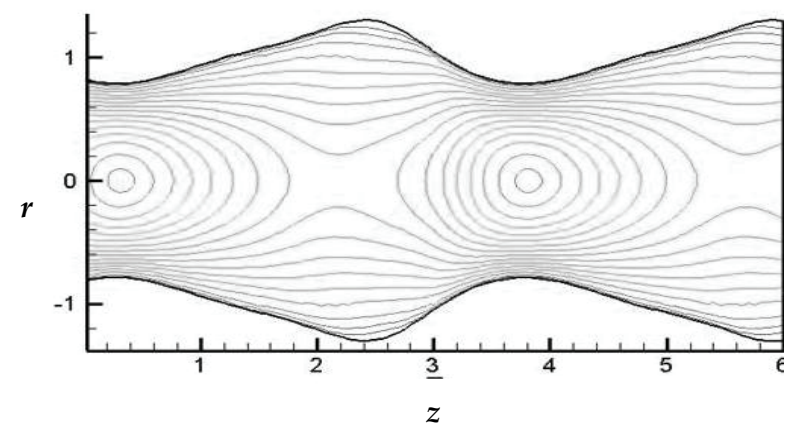

Fig. 9. Isovelocity lines for $\operatorname{Re}=1$ and $\varepsilon=0.3$. 


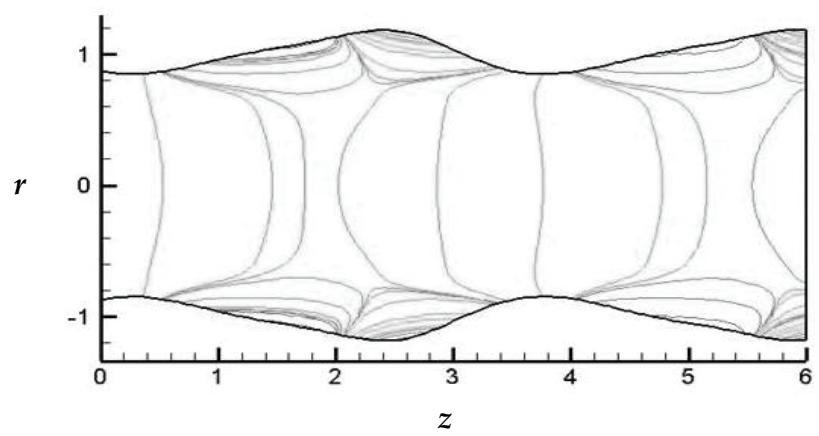

Fig. 10. Isobaric lines for $\operatorname{Re}=100$ and $\varepsilon=0.2$ from (42).

\subsubsection{Steady plastic flow in undulating channels}

A definition diagram for this flow is shown in fig. 11.

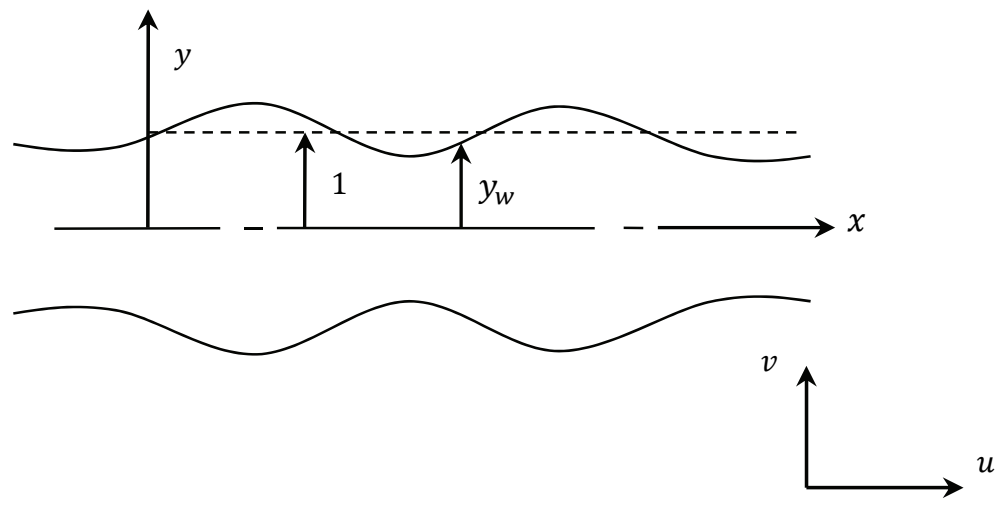

Fig. 11. Definition diagram for flow in undulating channels.

The Bingham constitutive equations are for this case are

$$
\begin{gathered}
\tau_{x x}=-\left(1-\frac{N}{I}\right) 2\left(\frac{\partial u}{\partial x}\right) \\
\tau_{y y}=-\left(1-\frac{N}{I}\right) 2\left(\frac{\partial v}{\partial y}\right) \\
\tau_{y x}=\tau_{x y}=-\left(1-\frac{N}{I}\right)\left(\frac{\partial u}{\partial y}+\frac{\partial v}{\partial x}\right)
\end{gathered}
$$

and the corresponding continuity and momentum equations are

$$
\frac{\partial u}{\partial x}+\frac{\partial v}{\partial y}=0
$$




$$
\begin{aligned}
& u \frac{\partial u}{\partial x}+v \frac{\partial u}{\partial y}=-\frac{1}{R e}\left(\frac{\partial \tau_{x x}}{\partial x}+\frac{\partial \tau_{x y}}{\partial y}\right)-\frac{1}{R e} \frac{\partial P}{\partial x} \\
& u \frac{\partial v}{\partial x}+v \frac{\partial v}{\partial y}=-\frac{1}{R e}\left(\frac{\partial \tau_{x y}}{\partial x}+\frac{\partial \tau_{y y}}{\partial y}\right)-\frac{1}{R e} \frac{\partial P}{\partial y}
\end{aligned}
$$

Next, the velocity, pressure and stream function are expanded as

$$
\begin{gathered}
u(x, y)=u_{0}(y)+\varepsilon u_{1}(x, y) \ldots \\
v(x, y)=\varepsilon v_{1}(x, y) \ldots \\
P(x, y)=P_{0}(x)+\varepsilon P_{1}(x, y) \ldots \\
\psi(x, y)=\psi_{0}(y)+\varepsilon \psi_{1}(x, y) \ldots \\
u(x, y)=\frac{\partial}{\partial y} \psi(x, y) \\
v(x, y)=-\frac{\partial}{\partial x} \psi(x, y) \\
\psi=\left(y-y_{w}\right)\left(g_{0}(y)+\varepsilon g_{1}(x, y)+\varepsilon^{2} g_{2}(x, y)+\cdots\right)
\end{gathered}
$$

where the wall is described by

$$
y_{w}=1+\varepsilon F(y) \sin (\omega x)
$$

Following a procedure similar to the one presented in section 3.2.1, it is found

$$
\begin{gathered}
\Psi_{0}=(y-1)^{2} g_{0}(y) \\
g_{0}(y)=-\frac{1}{6}(2 y+4-3 N) \\
\Psi_{1}=(1-y)^{2}\left(g_{1}(y) \sin (\omega x)+g_{2}(y) \cos (\omega x)\right)+2(1-y) F(y) \sin (\omega z) f_{0}(y)
\end{gathered}
$$

$F(y)$ is defined as $F=y$ and the first order stream function is modeled as

$$
\psi_{1}=A(y) \sin (\omega x)+B(y) \cos (\omega x)
$$

The unknown functions $A(y)$ and $B(y)$ are modeled as finite polynomial, ie

$$
\begin{aligned}
& A(y)=a_{0}+a_{1} y+a_{2} y^{2}+a_{3} y^{3}+a_{4} y^{4}+a_{5} y^{5}+a_{6} y^{6}+a_{7} y^{7}+a_{8} y^{8}+a_{9} y^{9} \\
& B(y)=b_{0}+b_{1} y+b_{2} y^{2}+b_{3} y^{3}+b_{4} y^{4}+b_{5} y^{5}+b_{6} y^{6}+b_{7} y^{7}+b_{8} y^{8}+b_{9} y^{9}
\end{aligned}
$$

The coefficients $a_{i}$ and $b_{j}$ are determined by substituting (75) and (76) in the equations for $\psi$ found from the momentum equations. In the following figures are presented plots of streamlines (equation (69)), isovelocity lines $\left(u^{2}+v^{2}=\right.$ const.), plug zones and axial velocity profiles

In figure 13 the plug zones are shown as shaded areas, which were determined by putting the condition that the shear stress should be equal or less the yield stress. The quasi-plug zones are zones where only $\tau_{r z} \leq 0$. 


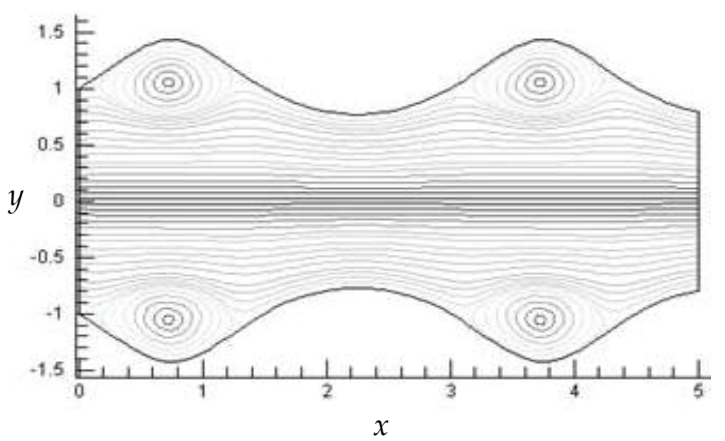

Fig. 12. Streamlines for $\mathrm{Re}=100, \mathrm{~N}=0.3$ and $\varepsilon=0.3$.

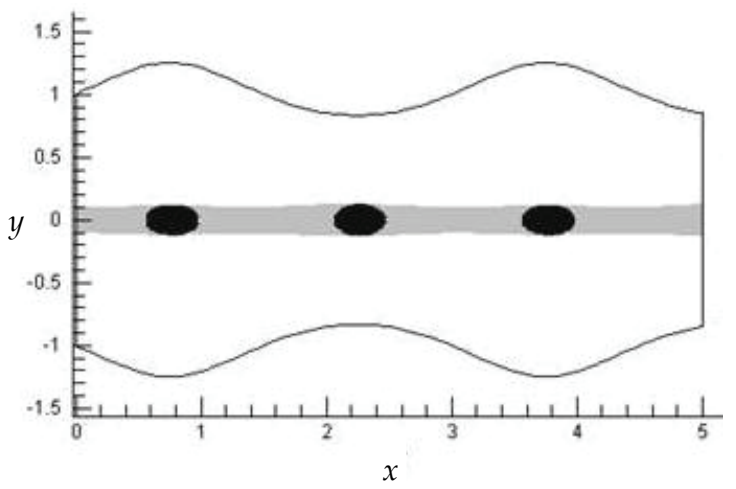

Fig. 13.Plug and quasi-plug zones for $R e=1, N=02$ and $\varepsilon=0.2$.

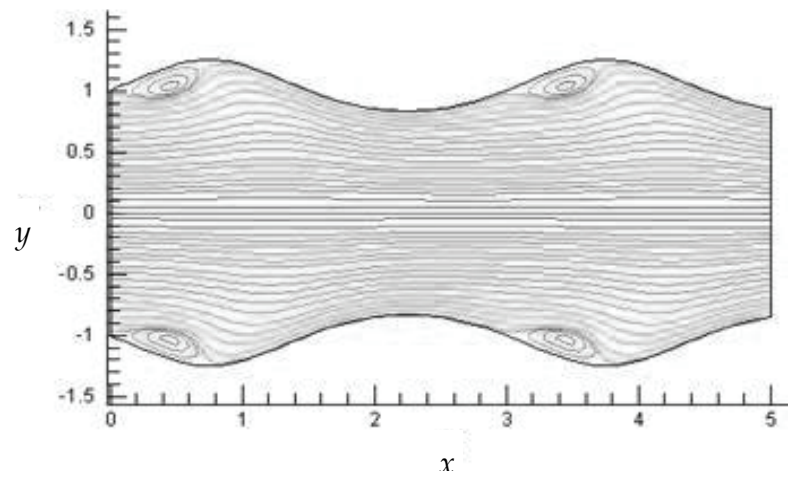

Fig. 14. Streamlines for $R e=20, N=0.1$ and $\varepsilon=0.2$. 


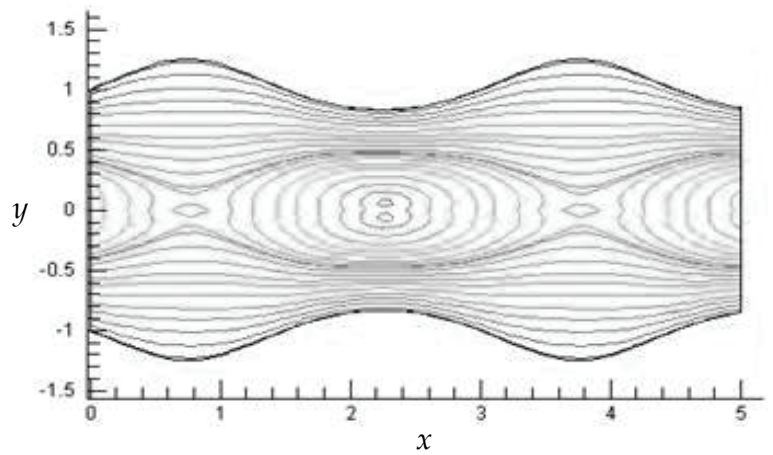

Fig. 15. Isovelocity lines for $R e=1, N=0.1$ and $\varepsilon=0.2$.

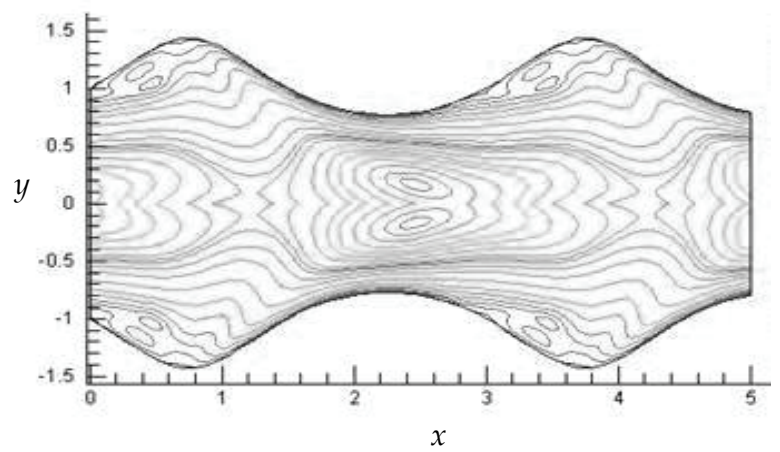

Fig. 16. Isovelocity lines for $R e=20, N=0.2$ and $\varepsilon=0.3$.

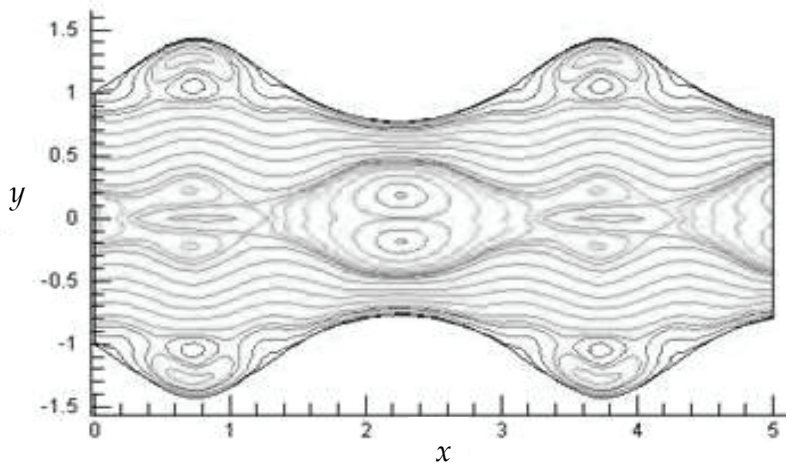

Fig. 17. Isovelocity lines for $R e=100, N=0.1$ and $\varepsilon=0.3$. 


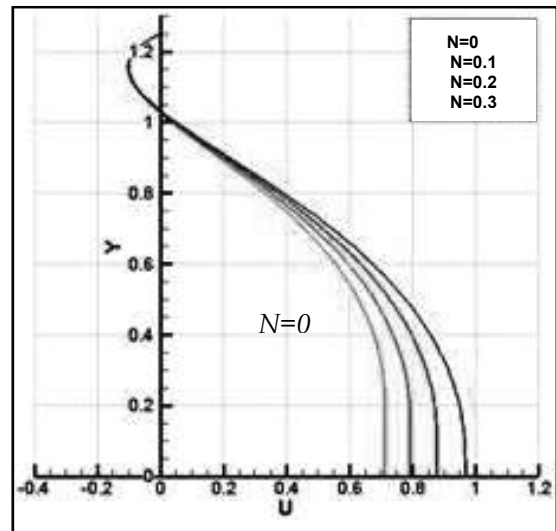

Fig. 18. Axial velocity profiles at $\mathrm{x}=0.75$ for $\varepsilon=0.2$ and $\operatorname{Re}=100$.

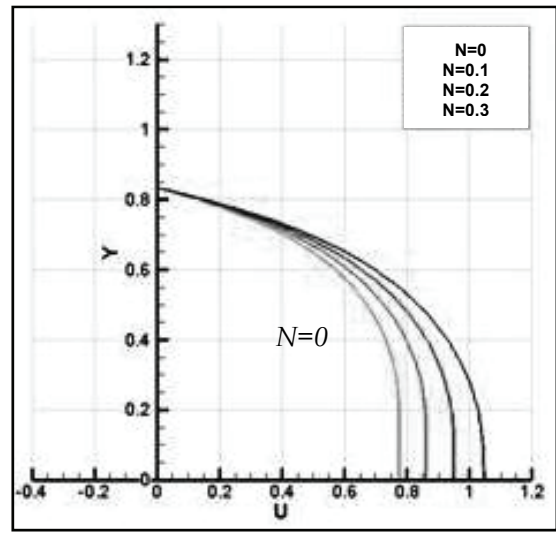

Fig. 19. Axial velocity profiles at $\mathrm{x}=2.25$ for $\varepsilon=0.2$ and $\operatorname{Re}=100$.

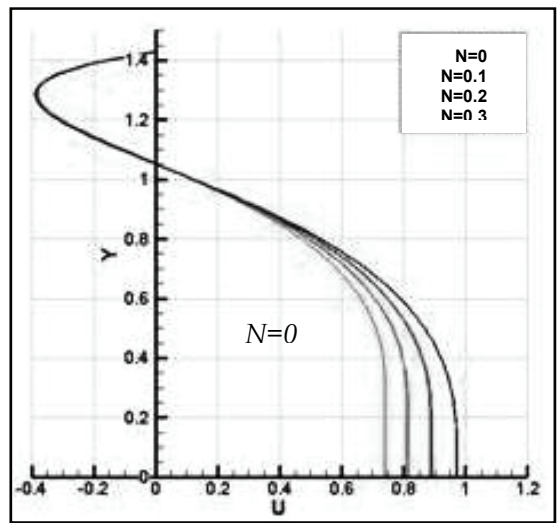

Fig. 20. Axial velocity profiles at $\mathrm{x}=0.75$ for $\varepsilon=0.3$ and $\operatorname{Re}=100$. 


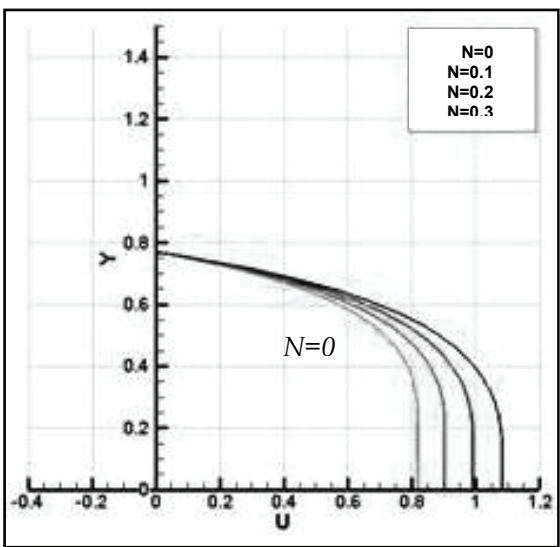

Fig. 21. Axial velocity profiles at $\mathrm{x}=2.25$ for $\varepsilon=0.3$ and $R e=100$.

\section{Conclusion}

The method here described can lead to very accurate solutions for the velocity field and related variables such as shear stress, rate of flow and pressure in a great variety of flows in tubes and channels. Symbolic software presently available, such Maple and MathCAD make it possible to obtain and compute higher order solutions that, in some cases, may have complex algebraic structures. The fact that for all cases here considered, ie cases where $n \geq$ $3, \varepsilon$ is much less than unity (cf table 2), leads to a regular perturbation scheme that in most cases requires terms up to second order to achieve enough accuracy. The cases when $n=1$ and $n=2$ deserve special mention. For $n=1$ the shape factor (1) describes an excentric circle, and for $n=2$ an ellipse. In this last instance $\varepsilon$ is not bounded and can take any finite value, which implies that the perturbation scheme would break down if $\varepsilon \geq 1$. So that, in this particular case, the method is limited to elliptical cross-sections of axes ratio close to unity. The method can be expanded to many more complex flow geometries. This possibility is implicit in the more general shape factor (3), which makes it necessary to develop a compound perturbation scheme, in terms of more than one perturbation parameter. The structure of the shape factor (1) determines that the analysis, especially for $n \geq 3$, is more sensible to the perturbation parameter for $r \geq 1$, ie close to the wall conduit. This requires a careful analysis of series convergency which should define the order of the higher order term considered. On the other hand, in the case of flow in straight tubes, in all cases studied, in a considerable region around the conduit axis, say for $r \leq 0.4$, the flow variables are independent of the boundary geometry and take the values of the corresponding flow in round tubes.

\section{Acknowledgment}

The authors acknowledge the financial support provided, at different stages of the work here presented, by FONDECYT-CONICYT and DICYT at the University of Santiago of Chile. 


\section{References}

Batchelor G. K. (2000). An Introduction to Fluid Dynamics. Cambridge University Press.

Cetin B. and Li D. (2008). Microfluidic continuous particle separation via ac-dielectrophoresis with $3 d$ electrodes. ASME International Mechanical Engineering Congress and Exposition.

Chen P.CH.; Wang H.; Park D.S.; Park S.; Nikitopoutos D.E.; Soper S. A. and Murphy M. C. (2008). Protein adsorption in a continuous flow micro-channel environment. ASME International Mechanical Engineering Congress and Exposition.

Forte J.A.; Sipahi R. and Ozturk A.(2008). A novel device for nonmagnetic particle navigation using ferrofluids manipulated by magnetic fields. ASME International Mechanical Engineering Congress and Exposition.

Gebauer P. and Bocek P.(2002). Recent progress in capillary isotachophoresis, pp. 3858-3864. Electrophoresis 23.

Letelier M.F. and Siginer D.A. (2003). Secondary Flows of Viscoelastic Liquids in Straight Tubes. pp. 5081-5095. International Journal of Solids and Structures, $\mathrm{N}^{\circ} 40$.

Letelier, M.F. and Siginer, D.A. (2007). On the Flow of a Class of Viscoinelastic-Viscoplastic Fluids in Tubes of Non-circular Contour, pp. 873 - 881. International Journal of Engineering Science, Vol. 45, Issue 11.

Letelier, M.F.; Leutheusser, H.J.; Member, ASCE, and Marquez Z., (1995). Laminar fluid transients in conduits of arbitrary cross section, pp.1069-1074. Journal of Engineering Mechanics.

Letelier, M.F.; Siginer D.A. and Cáceres, C.G. (2002). Pulsating Viscoelastic Flow in Tubes of Arbitrary Cross-Secction, Part I: Longitudinal Field. International Journal of NonLinear Mechanics.

Letelier, M.F.; Siginer, D.A.;. Stockle, J.S and Huilcan A. P. (2010). Laminar flow in tubes of circular cross section with arbitrary axial variation. ASME International Mechanical Engineering Congress and Exposition..

Mathies R.A. and Huang X.C.(1992). Capillary array electrophoresis: an approach to high-speed, high-throughput DNA sequencing pp. 167-169. Nature 359.

Pedley T. J. (2008). The Fluid Mechanics of Large Blood Vessels. Cambridge University Press.

Sommer G.J.; Chang D. S.; Jain A.; Langelier S. M.; Park S.; Rhee M.; Wang F.; Zeitun R. I. and Burns M. A. (2008). Introduction to microfluidics, pp.1- 33. In microfluidics for biological applications. W-C, Tian and E. Fine hout, editor, Springer.

Srivastava N.; Davenport RD.; Burns MA. (2005). Nanoliter viscometer for analyzing blood plasma and other liquid samples, pp. 383 - 392. Anal Chem. 77.

Stevenson, B. (2010). Análisis del campo de velocidades y de las condiciones de existencia y características de la zona tapón en flujos plásticos en ductos de secciones arbitrarias. Mechanical Engineering thesis, Master degree, University of Santiago of Chile.

Svensson, P.J. (2010). Análisis de la zona tapón en flujo plástico en canales ondulados. Mechanical Engineering thesis, University of Santiago of Chile.

Woolley A. and Mathies R. (1994). Ultra-high-speed and fragment separations using microfabricated capillary array electrophoresis chips, pp. 11348 - 11352. Proceedings of the National Academy of Sciences 91. 
Xue, S.C.; Phan-Thien N. and Tanner, R.I. (1995). Numerical study of secondary flows of viscoelastic fluid in straight pipes by an implicit finite volume method, pp. 191-213. Journal of Non-Newtonian Fluid Mechanics, 59.

Yager P.; Edwards T.; Fu E.; Helton K.; Nelson K.; Tam MR.; Weigl BH.( 2006) Microfluidic diagnostic technologies for global public health, pp.412 - 418. Nature 442. 


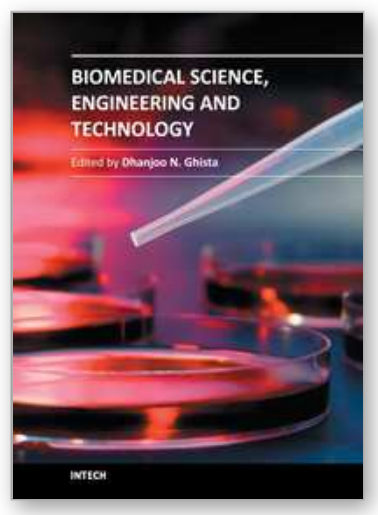

\author{
Biomedical Science, Engineering and Technology \\ Edited by Prof. Dhanjoo N. Ghista
}

ISBN 978-953-307-471-9

Hard cover, 902 pages

Publisher InTech

Published online 20, January, 2012

Published in print edition January, 2012

This innovative book integrates the disciplines of biomedical science, biomedical engineering, biotechnology, physiological engineering, and hospital management technology. Herein, Biomedical science covers topics on disease pathways, models and treatment mechanisms, and the roles of red palm oil and phytomedicinal plants in reducing HIV and diabetes complications by enhancing antioxidant activity. Biomedical engineering coves topics of biomaterials (biodegradable polymers and magnetic nanomaterials), coronary stents, contact lenses, modelling of flows through tubes of varying cross-section, heart rate variability analysis of diabetic neuropathy, and EEG analysis in brain function assessment. Biotechnology covers the topics of hydrophobic interaction chromatography, protein scaffolds engineering, liposomes for construction of vaccines, induced pluripotent stem cells to fix genetic diseases by regenerative approaches, polymeric drug conjugates for improving the efficacy of anticancer drugs, and genetic modification of animals for agricultural use. Physiological engineering deals with mathematical modelling of physiological (cardiac, lung ventilation, glucose regulation) systems and formulation of indices for medical assessment (such as cardiac contractility, lung disease status, and diabetes risk). Finally, Hospital management science and technology involves the application of both biomedical engineering and industrial engineering for cost-effective operation of a hospital.

\title{
How to reference
}

In order to correctly reference this scholarly work, feel free to copy and paste the following:

Mario F. Letelier and Juan S. Stockle (2012). A Shape-Factor Method for Modeling Parallel and Axially-Varying Flow in Tubes and Channels of Complex Cross-Section Shapes, Biomedical Science, Engineering and Technology, Prof. Dhanjoo N. Ghista (Ed.), ISBN: 978-953-307-471-9, InTech, Available from: http://www.intechopen.com/books/biomedical-science-engineering-and-technology/a-shape-factor-method-formodeling-parallel-and-axially-varying-flow-in-tubes-and-channels-of-comple

\section{INTECH}

open science | open minds

\section{InTech Europe}

University Campus STeP Ri

Slavka Krautzeka 83/A

51000 Rijeka, Croatia

Phone: +385 (51) 770447

Fax: +385 (51) 686166

\section{InTech China}

Unit 405, Office Block, Hotel Equatorial Shanghai

No.65, Yan An Road (West), Shanghai, 200040, China 中国上海市延安西路65号上海国际贵都大饭店办公楼405单元

Phone: +86-21-62489820

Fax: $+86-21-62489821$ 
www.intechopen.com 
(C) 2012 The Author(s). Licensee IntechOpen. This is an open access article distributed under the terms of the Creative Commons Attribution 3.0 License, which permits unrestricted use, distribution, and reproduction in any medium, provided the original work is properly cited. 